\title{
Satisfaction with life in a group of psoriasis patients
}

\author{
Barbara Jankowiak ${ }^{1,2}$, Sylwia Sekmistrz², Beata Kowalewska ${ }^{1,2}$, Wiaczesław Niczyporuk ${ }^{2}$, Elżbieta Krajewska-Kułak \\ 1Department of Integrated Medical Care, Medical University of Bialystok, Poland \\ Head: Prof. Elżbieta Krajewska-Kułak MD, PhD \\ 2Medical Institute of Medicine, The State College of Computer Science and Business Administration, Lomza, Poland \\ Head: Barbara Jankowiak MD, PhD
}

Postep Derm Alergol 2013; XXX, 2: 85-90

DOI: $10.5114 / p d i a .2013 .34156$

\begin{abstract}
Introduction: Psoriasis is one of the most frequent inflammatory diseases of the skin, associated with an epidermal proliferation and a specific morphology of lesions. Patients with psoriasis perceive their appearance specifically; they are frequently rejected by their surroundings and perceive their quality of life as considerably poorer. Aim: To evaluate the satisfaction with life in patients with psoriasis, and to analyze the effect of this disease on the prevalence of depression in this group.

Material and methods: The study included 100 psoriasis vulgaris patients treated at the Voivodeship Outpatient Clinic of Skin and Venereal Diseases in Lomza (Poland). Sociodemographic data of the participants and the clinical characteristics of the disease were collected using a standardized questionnaire survey. The global feeling of satisfaction with life was evaluated with the Satisfaction with Life Scale and the Beck's Depression Inventory.

Results: Mean SWLS scores suggested that the examined patients experienced moderate levels of satisfaction with life (18.92 and 18.69 points in women and men, respectively). The life satisfaction was the highest amongst patients between 50 and 60 years of age $(p=0.81)$. The mean score of the Beck Depression Inventory was at a threshold of mild depression (14.08 and 13.65 points in women and men, respectively).

Conclusions: Our participants presented moderate levels of satisfaction with life. A lower satisfaction with life was associated with a poorer quality of life and a higher prevalence of depressive symptoms.
\end{abstract}

Key words: psoriasis, satisfaction with life, quality of life.

\section{Introduction}

Psoriasis is one of the most frequent diseases of the skin. Its average prevalence is estimated at 1-3\% [1]. Although the clinical diagnosis of the disease is not difficult, some components of its pathogenesis, unexpected manifestation, clinical response to therapy, as well as association with other conditions, are still not understood. Currently, a wide choice of available treatment modalities can markedly reduce or nearly eliminate the symptoms of psoriasis, improve skin condition, and attenuate local symptoms [1-5]. However, individuals with psoriasis usually begin their therapy without motivation and do not feel well until they have found proper balance between the treatment benefits and risks.

Individuals with dermatological conditions perceive their image specifically, due to disturbed esthetic, as well as disrupted communicatory and perceptive functions of the skin. The patients experience the feeling of stigma: they are fre- quently rejected by their social surroundings and perceive their quality of life as markedly lower. All of these conditions can considerably modulate the mental status of an individual who is affected by such an unexpected disorder [3, 6-8].

\section{Aim}

The aim of this study was to evaluate the satisfaction with life in patients with psoriasis, and to analyze the effect of this disease on the prevalence of depression in this group.

\section{Material and methods}

The study included 100 individuals with confirmed psoriasis vulgaris, all treated at the Voivodeship Outpatient Clinic of Skin and Venereal Diseases in Lomza (Poland). Patients with diseases other than psoriasis vulagris were eliminated. The severity of psoriasis was 
assessed by PASI (Psoriasis Area and Severity Index). Mean PASI score was $\leq 20$ (mild to moderate psoriasis). This group included $64 \%$ of women and $36 \%$ of men.

The survey was based on a standardized interview collected with a questionnaire developed solely for the purpose of this study. The sociodemographic data of participants (gender, age, education, place of residence, marital status, professional activity) as well as the clinical characteristics of the disease (duration, familial history, location of lesions, most harmful symptoms, and the influence on the quality of life) were recorded.

The global feeling of satisfaction with life was evaluated using the Satisfaction with Life Scale (SWLS) by Diener et al. [9], in the Polish adaptation by Juczyński [10]. SWLS was developed for individual and group examination of healthy and diseased adults. This scale includes five statements. A participant scores each statement with regards to its consistency with his/her entire life. The scale ranges between 1 and 7, with 1 corresponding to "I completely disagree", and 7 - "I absolutely agree". The reliability of the Polish version of SWLS is high (the $\alpha$ Cronbach's index $=0.81$ ). The overall score expresses the global level of satisfaction with life which can range between 5 and 35 points. Higher scores suggest a greater satisfaction with life.

Furthermore, the participants completed the Polish version of the Beck Depression Inventory, used to detect depressive symptoms and grade their severity [11].

The test includes 21 items pertaining to various symptoms of depression. The respondents were asked to indicate one out of four answers being the most consistent with their status in the past 30 days. The answers were scored between 0 and 3 points. Consequently, the minimal overall score of the test was 0 , and maximal score was 63. In practice, however, results above 50 points are not obtained since patients with such an advanced depression are unable to complete the questionnaire by themselves. The results of the test are interpreted as follows: 0-11 points: lack of depression; 12-19 points: mild depression; 20-25 points: moderate depression; above 26 points: severe depression.

The protocol of the study was approved by the Local Bioethical Committee of the Medical University in Bialystok (R-I-002-375/2011).

\section{Statistical analysis}

Mean values were compared with the Student $t$-test. The results were expressed as $p$-values, referred to as the test's probability or critical level of significance. A difference was considered significant whenever $p<0.05$.

\section{Results}

The studied group $(n=100)$ included countryside (29\%) and city dwellers (71\%). The age of respondents ranged between 16 and 71 years. This age category predominated in a subgroup of women as well (32.8\%), while the most prevalent male age category included individuals above 60 years of age $(27.8 \%)$. Most of the patients were married (55\%). The fraction of participants who had completed a higher level of education was the most prevalent amongst women (48.4\%), while individuals who had completed a professional education predominated amongst men (36.1\%). Most participants were white-collar workers (42\%) or performed physical work (32\%). Overall, old-age pensioners corresponded to nearly $7 \%$ of all participants.

In most patients, the first episode of psoriasis occurred when they were of less than 20 years of age (48\%); 33\% of the participants were diagnosed with the disease between 20 and 29 years, $14 \%$ - at 30-39 years of age, and $5 \%$ above 40 years. More than $50 \%$ of respondents reported the familial prevalence of psoriasis. In the case of nearly $22 \%$ of participating women, their mothers were equally affected, whereas in men, fathers were the most frequent relatives affected by psoriasis (19.4\%).

The most frequently declared locations of psoriatic lesions included elbows (34.4\% of women and $27.8 \%$ of men), knees ( $26.6 \%$ of women and $22.2 \%$ of men), back (10.9\% of women and $16.7 \%$ of men), and scalp (18.8\% of women and $19.4 \%$ of men). Furthermore, the respondents comprehensively reported on the most embarrassing symptoms of psoriasis. The most frequently reported symptoms included itching (52\%), peeling of the skin (32\%), burning (14\%), skin dryness (7\%), and unattractive appearance $(6 \%)$.

Women and men differed slightly in terms of the mean overall SWLS scores. In both genders, the mean values corresponded to moderate satisfaction with life and were at a median level of all available points. The overall scores in women and men were 18.92 and 18.69, respectively.

The satisfaction of life was highest amongst participants aged 50 and 60 years, regardless of the sex. Taking into account the average SWLS score in the entire sample, the satisfaction was the lowest amongst patients aged 30 to 39 years. When participants' gender was considered, the scores were lowest amongst women above 60 years of age and in 40-49-year-old men (Table 1).

The results were surprising when the duration of the disease was considered (Figure 1). A longer duration of the disease was associated with a higher satisfaction with life. This phenomenon was most evident in women. In contrast, in men the disease lasting longer than 40 years was reflected by a marked decrease in the satisfaction levels, despite men having an increased satisfaction with life proportionally to the disease duration up to this cutoff value.

Additionally, the data obtained with the Beck Depression Inventory were analyzed in order to analyze the prevalence and severity of depressive symptoms. The respondents were asked to indicate one out of four answers to 21 questions included in the inventory, being 
Table 1. Mean SWLS scores by participants' gender and age

\begin{tabular}{|c|c|c|c|c|}
\hline \multirow{2}{*}{$\begin{array}{l}\text { Age category } \\
\text { [years] }\end{array}$} & \multicolumn{2}{|c|}{ Mean } & \multirow{2}{*}{$\begin{array}{l}\text { Student } \\
t \text {-test }\end{array}$} & \multirow{2}{*}{$\begin{array}{l}\text { Mean } \\
\text { overal }\end{array}$} \\
\hline & Women & Men & & \\
\hline$<20$ & - & 19.25 & - & 19.25 \\
\hline $20-29$ & 20.67 & 16.67 & $p=0.36$ & 19.94 \\
\hline $30-39$ & 17.33 & 18.80 & $p=0.63$ & 17.62 \\
\hline $40-49$ & 18.41 & 15.71 & $p=0.31$ & 17.63 \\
\hline $50-60$ & 21.13 & 20.43 & $p=0.81$ & 20.80 \\
\hline$>60$ & 17.00 & 19.90 & $p=0.40$ & 19.64 \\
\hline
\end{tabular}

the most consistent with their state in the last 30 days. The answers were scored between 0 and 3 points. The mean score of the respondents was at a threshold of mild depression since it amounted to $\approx 14$ points (14.08 and 13.65 in women and men, respectively).

Women and men differed slightly in terms of the mean scores obtained using the discussed questionnaire (Table 2). In women, the scores were between 12 and 15 points irrespective of the age category, which corresponded to a mild depression. In the case of males up to 39 years of age, the average score ranged between 7 and 9 points, suggesting the lack of depression, while a mild depression was diagnosed in individuals above 40 years (mean score 15-18 points).

The respondents were asked to answer the question: "Did the disease diminish your quality of life?". Most of the patients (61\%) answered this question affirmatively; the negative answer was given by $39 \%$ of the respondents. Noticeably, men seem more indifferent to the disease since nearly half of them (47.2\%) did not declare it affected their quality of life, as compared to only $34.4 \%$ of women.

The participants who answered the aforementioned question affirmatively were asked to answer another one: "Which aspect of your life was most severely affected by the disease?". The answers were more comprehensive in the case of women who mentioned changes in the style of life (53.1\%), worse appearance (34.4\%), poorer economic situation (23.4\%), avoiding contacts with other people (20.3\%), lower self-evaluation (15.6\%), limitations in professional activity (14.1\%), diminished mood (14.1\%), shame (7.8\%), and embarrassment (4.7\%). In turn, men pointed to the fear of sexual contacts (72.4\%), relationships with women (50\%), going to the beach, swimming pool, or gym (44.4\%), shame (19.4\%), and avoiding other people $(16.7 \%)$. In the opinion of $18.8 \%$ of women and $5.6 \%$ of men, psoriasis played a role in the break-up of a relationship with their partners.

\section{Discussion}

Psoriasis is one of the most frequent dermatological conditions. It is characterized by periodical recurrences and

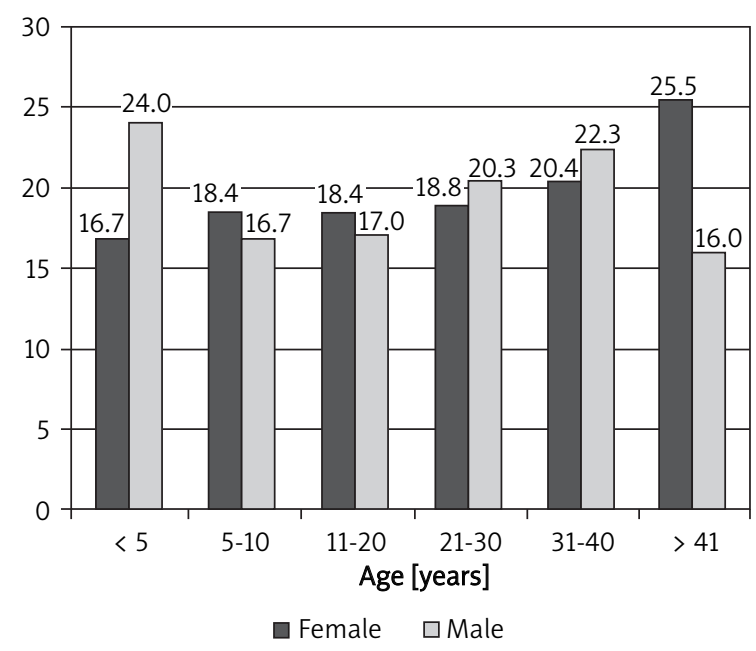

Figure 1. Duration of the disease and the satisfaction with life scores (SWLS) depending on participant's gender

Table 2. Mean scores of the Beck Depression Inventory by participants' gender and age

\begin{tabular}{lcccc}
\hline $\begin{array}{l}\text { Age category } \\
\text { [years] }\end{array}$ & \multicolumn{2}{c}{ Mean } & $\begin{array}{c}\text { Student } \\
\text { t-test }\end{array}$ & $\begin{array}{c}\text { Mean } \\
\text { overall }\end{array}$ \\
\cline { 2 - 3 } Women & Men & & \\
\hline$<20$ & - & 9 & - & 9 \\
\hline $20-29$ & 12 & 9 & $p=0.64$ & 10 \\
\hline $30-39$ & 14 & 7 & $p=0.08$ & 10 \\
\hline $40-49$ & 15 & 16 & $p=0.87$ & 15 \\
\hline $50-60$ & 15 & 15 & $p=1.00$ & 15 \\
\hline$>60$ & 13 & 18 & $p=0.27$ & 15 \\
\hline
\end{tabular}

remissions. The disease can manifest at any age, but most frequently it develops in individuals under 40 years of age. Two types of psoriasis can be distinguished using the age criterion. Type I psoriasis manifests at a younger age, before 40 years of age. In some cases its manifestation could be quite severe and recurrence is a frequent finding. Type II psoriasis manifests at an older age, with a peak incidence at about 60 years of age. Usually, this type of psoriasis has no familial pattern. The manifestation of the disease is relatively stable and the lesions are milder [1, 12-14].

In $48 \%$ of our respondents, psoriasis manifested as early as before they turned 20 , and in another $33 \%$, it developed at 20-29 years. These data suggest a possible predominance of type I psoriasis.

The clinical manifestation of the disease can be variable - from single laminar lesions, plaque psoriasis of the scalp and larger areas of body, to a generalized involvement of the entire skin (erythrodermic psoriasis) $[1,12-14]$.

The respondents declared itching and peeling of the skin as the most harmful psoriatic symptoms. Unattrac- 
tive appearance was another frequently reported problem, being a consequence of the disease that severely affected the patient's life. We have revealed a relationship between the perceived physical discomfort and the quality of life $(p=0.00015)$. The patients who reported more physical symptoms usually declared a larger impairment of their quality of life.

According to many authors, skin diseases can markedly affect all areas of life - professional and social activities, as well and social and sexual life $[3,7]$. Many previous studies analyzed the effects of various factors on the quality of life in patients with psoriasis [15-20]. The results of the quality of life survey conducted by the National Psoriasis Foundation suggests that living with psoriasis can be more harmful than in the case of many other chronic conditions including coronary heart disease and chronic obstructive pulmonary disease (COPD). In this study, psoriasis was ranked the second most devastating disease, following depression [3].

Our study results' analysis suggests that most patients (61\%) perceive some negative effects of the disease on the quality of their lives. In this study, individuals with a shorter history of psoriasis (up to 15 years) perceived their quality of life to be poorer than those with a longer duration of the condition (more than 25 years; $p=0.0304$ ). Furthermore, this sphere was scored poorly by women (65.6\%) as compared to men $(52.8 \%$, $p=0.4189$ ), as well as by individuals with higher education as compared to those with secondary and professional education ( $p=0.0735)$.

Frequently, patients with psoriasis perceive themselves as socially excluded; they do not accept their body image, and feel tired with the disease-related discomfort and the lack of acceptance from their peers. This usually leads to a gradual separation and social withdrawal. The psoriatic foci are visible to the others and thus can raise social intolerance. Frequently, healthy individuals consider psoriasis to be a contagious condition, and the resultant avoidance of physical contact enhances the feeling of rejection and indignity amongst the patients. Due to external signs of the disease, patients with psoriasis avoid swimming and sunbathing, and are reluctant to use public means of transportation or to go to the hairdresser [7, 21-24].

Good mood is an important component of health, and not infrequently is even equated to the latter. Good temper is an important determinant of one's mood. If patients are able to keep a good mood and feel comfortable with themselves and their surroundings, they usually will not perceive their disease negatively. In contrast, people unable to maintain a good mood who are sensitive to the negative psychological effects of the disease will perceive the latter as a considerable burden. Generally, patients living in harmony with themselves and their surroundings, having satisfactory jobs as well as support and understanding from their families, colleagues, and friends, will find more strength to cope with the episodes of the disease exacerbation [25-27].

According to the literature, the level of everyday functioning in psoriasis patients is markedly lower than in healthy individuals. Feelings of stigma, vulnerability to others' opinions, anticipation of rejection, and avoidance of social contacts modulate the quality of the patient's life considerably and promote the appearance of depressive symptoms, as confirmed by the results of this study $[25,28,29]$. Shaking hands or touching the skin of the patient with psoriasis can exert a therapeutic effect as it corresponds to the acceptance and understanding of the disease. In contrast, poor mood, and the feeling of rejection can diminish the overall quality of life score.

A subjective feeling of good mood is determined by three factors: the level of satisfaction with life, positive feelings, and the lack of the negative feelings. The level of self-perceived satisfaction with life results from comparing the present situation experienced by an individual to him/herself defined standard. If the result of this comparison proves satisfactory, one will feel satisfied with his/her life. The SWLS-determined level of satisfaction with life corresponds to the feeling of satisfaction with one's achievements and conditions [16]. Using this scale in our responders revealed their moderate satisfaction with life. The average score of women was 18.92, being slightly higher than in men (18.69). Surprisingly, the satisfaction with life increased with the duration of the disease. Perhaps, this phenomenon resulted from accepting the disease and enjoying life irrespective of this condition.

Familial status can modulate both mental status and life comfort. Nonetheless, family members should provide the patient with understanding and support since any stress can exacerbate the recurrence of psoriasis or delay its remission. Each patient requires much support from his/her relatives. This is equally important in individuals with large, visible lesions and in those with small, occult disease foci. In most cases, there was a correlation between having a family and the satisfaction with life and depression levels. Indeed, in this study, $67 \%$ of married women and $56 \%$ of married men scored their satisfaction with life at an average level of 28 points; the corresponding depression scores were 11 and 9 points for women and men, respectively.

Aging is associated with a diminished physical and mental capacity and a co-existence of various disorders. As a result, both comfort and satisfaction with life are reported to decrease gradually with one's age. In our study, however, younger and middle-aged individuals proved to be the ones who more frequently experienced discomfort in their everyday lives. This finding suggests that in some cases, symptoms of psoriasis can be reflected by serious social problems, which is followed by a gradual adaptation occurring along with the progression of the disease (usually after approximately 10 years). 
Diseases of the skin are particularly devastating to patient's psychology since they can be seen by others. Many individuals feel less attractive if their skin is red and peeling. Some are even abhorred with themselves, and experience shame and guilt [25, 28-32]. The examination of our psoriasis patients using Beck's Depression Inventory revealed a mild depressive status. Although in both genders the average scores fit into a mild depression category, the average score in women was slightly higher than in men (14.08 vs. 13.65).

Results by Devrimci-Ozguven et al. show that there was a much greater degree of depression in patients with psoriasis. In addition, the risk of developing psoriasis significantly increased in patients with moderate to severe depression. The results suggest that there is an association between psoriasis and mental health problems and further research is required [33].

The analysis of the association between a disease and the quality of life is vital for the proper assessment of therapeutic activities. Determination of health-related quality of life enables selection of such a treatment modality that offers optimal therapeutic effects. Furthermore, it facilitates communication between the physician and the patient and improves the course of therapy and medical care. Unfortunately, the quality of life is usually not measured in individual psoriasis patients; in most cases the assessment is limited to a few questions dealing with one's mood and coping with the disease. Usually, such an approach is insufficient as many potentially impaired aspects of life are not considered. In contrast, a routine quality of life assessment can influence both the selection and the effectiveness of therapy. Furthermore, it motivates the patients to self-control and to follow medical advice and prescriptions, and shortens time required for them to understand their threats and problems, facilitating their referral to a specialist.

\section{Conclusions}

Psoriasis has a considerable negative impact on patients' quality of life, as confirmed by $61 \%$ of participants of this study. Overall, the quality of life scores were slightly lower in women than in men. Our participants presented moderate levels of satisfaction with life, corresponding to a median level of points available in the used scale. Most of our respondents represented a mild form of depression ( $\approx 14$ points), with the latter being slightly more prevalent in women than in men. A lower satisfaction with life was associated with poorer quality of life and a higher prevalence of depressive symptoms.

\section{References}

1. Langley RG, Krueger GG, Griffiths CE. Psoriasis: epidemiology, clinical features, and quality of life. Ann Rheum Dis 2005, 64: ii18-23.
2. Griffiths CE, Barker JN. Pathogenesis and clinical features of psoriasis. Lancet 2007; 370: 263-71.

3. Krueger G, Koo J, Lebwohl M, et al. The impact of psoriasis on quality of life: results of a 1998 National Psoriasis Foundation patient-membership survey. Arch Dermatol 2001; 137: 280-4.

4. Zejda JE, Brożek GM, Farnik M, Smółka I. Social and familyrelated correlates of medical care utilization by asthmatic children in Upper Silesia, Poland. Ann Agric Environ Med 2012; 19: 141-5.

5. Rasmy H, Mikhael N, Ismail S. Interleukin-18 expression and the response to treatment in patients with psoriasis. Arch Med Sci 2011; 7: 713-9.

6. Finkelstein J, Lapshin O, Wasserman E. Randomized study of different anti-stigma media. Patient Educ Couns 2008; 71: 204-14.

7. Gelfand J, Feldman S, Stern R, et al. Determinants of quality of life patients with psoriasis: a study from the U.S. population. J Am Acad Dermatol 2004; 51: 704-8.

8. Evers AW, Lu Y, Duller P, et al. Common burden of chronic skin diseases? Contributors to psychological distress in adults with psoriasis and atopic dermatitis. Br J Dermatol 2005; 152: 1275-81.

9. Diener E, Emmons RA, Larson RJ, et al. The satisfaction with life scale. J Pers Assess 1985; 49: 71-8.

10. Juczyński Z. Measurement tools, promotion and health psychology. Pracownia Testów Psychologicznych PTP, Warsaw 2009; 128-36.

11. Zawadzki B, Popiel A, Praglowsck E. Psychometric properties of the Polish version of the Aaron T. Beck's Depression Inventory BDI-II [Polish]. Psychologia Etologia Genetyka 2009; 19: 71-95.

12. Christophers E. Psoriasis - epidemiology and clinical spectrum. Clin Exp Dermatol 2001; 26: 314-20.

13. Elder JT, Nair RP, Voorhees JJ. Epidemiology and the genesis of psoriasis. J Invest Dermatol 1994; 102: 24S-7S.

14. Naldi L. Psoriasis. Dermatol Clin 1995; 13: 635-47.

15. Chren MM, Lasek RJ, Quinn LM, et al. Convergent and discriminant validity of a generic and a disease-specific instrument to measure quality of life in patients with skin disease. J Invest Dermatol 1997; 108: 103-7.

16. De Korte J, Mombers FM, Sprangers MA, et al. The suitability of quality-of-life questionnaires for psoriasis research: a systematic literature review. Arch Dermatol 2002; 138: 1221-7.

17. Koo J. Population - based epidemiologic study of psoriasis with emphasis on quality of life assessment. Dermatol Clin 1996; 14: 485-96.

18. Weiss SC, Kimball AB, Liewehr DJ, et al. Quantifying the harmful effect of psoriasis on health-related quality of life. J Am Acad Dermatol 2002; 47: 512-8.

19. Zachariae R, Zachariae C, Ibsen H, et al. Dermatology life quality index: data from Danish inpatients and outpatients. Acta Derm Venereol 2000; 80: 272-6.

20. Kimball AB, Jacobson C, Weiss S, et al. The psychosocial burden of psoriasis. Am J Clin Dermatol 2005; 6: 383-92.

21. Jankowiak B, Krajewska-Kułak E, Baranowska A, et al. The importance of the health education in life quality improvement in patients with psoriasis. Ann Acad Med Bialostocennsis 2005; 50 Supl. 1: 145-7.

22. Eghlileb AM, Davies EE, Finlay AY. Psoriasis has a major secondary impact on the lives of family members and partners. Br J Dermatol 2007; 156: 1245-50. 
23. Richards HL, Fortune DG, Griffiths CE, et al. The contribution of perceptions of stigmatization to disability in patients with psoriasis. J Psychosom Res 2001; 50: 11-5.

24. Jenner N, Campbell J, Plunkett A, et al. Cost of psoriasis: a study on the morbidity and financial effects of having psoriasis in Australia. Australas I Dermatol 2002; 43: 255-61.

25. Ginsburg IH. Psychological and psychophysiological aspects of psoriasis. Dermatol Clin 1995; 13: 793-804.

26. Zachariae R, Oster H, Bjerring P, et al. Effects of psychologic intervention on psoriasis: a preliminary report. J Am Acad Dermatol 1996, 34: 1008-15.

27. de Arruda LH, De Moraes AP. The impact of psoriasis on quality of life. Br J Dermatol 2001; Suppl. 58: 33-6.

28. Sharma N, Koranne RV, Singh RK. Psychiatric morbidity in psoriasis and vitiligo: a comparative study. J Dermatol 2001; 28: 419-23.

29. Gupta MA, Gupta AK. Depression and suicidal ideation in dermatology patients with acne, alopecia areata, atopic dermatitis and psoriasis. Br J Dermatol 1998; 138: 846-50.

30. Burr S, Gradwell C. The psychosocial effects of skin diseases: need for support groups. Br I Nurs 1996; 19: 1177-82.

31. Rapp SR, Cottrell CA, Leary MR. Social coping strategies associated with quality of life decrements among psoriasis patients. Br J Dermatol 2001; 145: 610-6.

32. Weiss SC, Kimball AB, Liewehr DJ, et al. Quantifying the harmful effect of psoriasis on health-related quality of life. J Am Acad Dermatol 2002; 47: 512-8.

33. Devrimci-Ozguven H, Kundakci TN, Kumbasar H, et al. The depression, anxiety, life satisfaction and affective expression levels in psoriasis patients. J Eur Acad Dermatol Venereol 2000; 14: 267-71. 